\title{
High Temperature Superconducting Current Leads for the Large Hadron Collider
}

\author{
A.Ballarino \\ CERN, European Organization for Nuclear Research, 1211 Geneva 23, Switzerland
}

\begin{abstract}
The Large Hadron Collider (LHC) will be equipped with about 8000 superconducting magnets. Some 3380 leads will feed the currents ranging from 60 to $13000 \mathrm{~A}$. To reduce the heat inleak into the liquid helium, CERN aims to use High Temperature Superconducting material for leads having current ratings between 600 and $13000 \mathrm{~A}$. Specifications have been written for 13000 A current leads, incorporating a High Temperature Superconducting section, for the main magnets of the LHC, and contracts have been placed with several firms for the supply of prototypes for comparative testing. The leads used for feeding locally the 60 and $120 \mathrm{~A}$ dipole orbit correctors will be conventional conduction cooled resistive leads. An optimized lead of variable cross section has been tested, and an integral design has been initiated. This report describes the design status of the current leads for the LHC, emphasizing, for the different solutions, the principle of optimization and the choice of cooling methods.
\end{abstract}

\section{INTRODUCTION}

The powering of the Large Hadron Collider superconducting magnets will require about 3380 current leads, with current ranging from 60 to $13000 \mathrm{~A}$. The total current to be transported inside and outside the cold mass amounts to about 3.6 MA and is distributed among the different ratings as shown in Fig. 1. Conventional vapour cooled leads would create a total heat load into the liquid helium of about $4 \mathrm{~kW}$. The use of HTS material, replacing the conventional metal in the coldest part of the lead, produces a significant reduction of heat load, with important savings in liquefaction power with respect to conventional leads.

Following encouraging tests of HTS samples carrying currents ranging from 600 to $12500 \mathrm{~A}, \mathrm{CERN}$ aims to apply High Temperature Superconducting technology to all the leads operating at these current levels. The corrector leads, thanks to the low current rating, introduce tolerable heat inleaks if made with a conventional metal carefully designed to minimise the heat load into the cryogenic system.

\section{HTS MATERIAL TESTING}

A series of tests has been performed to check the viability of HTS components in high-current leads [1], [2], [3].

Manuscript received September 14, 1998.

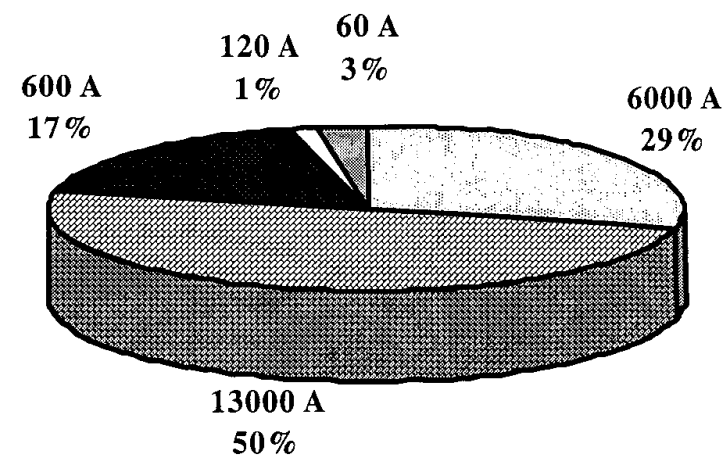

Fig. 1. Current ratings of the LHC current leads.

Different samples of HTS material (BSCCO Ag-Au tapes, YBCO modules, YBCO bars and BSCCO tubes), optimised to carry $12500 \mathrm{~A}$ at about $70 \mathrm{~K}$, have been tested in a test facility designed and built by Oxford Instruments in close collaboration with CERN.

The results have shown that all these materials are able to conduct high current with low heat conduction. The limitations encountered have been high contact resistance at the two ends of the superconductor and possible breakage of bulk material due to localised heating during quench.

To investigate this second problem, additional tests have been performed on two bulk samples, both protected with a metallic cladding to assist the process of quench propagation. The samples have been designed to carry $12000 \mathrm{~A}$,. in the insulation vacuum of the cryostat, between terminals at $4.2 \mathrm{~K}$ and $70 \pm 5 \mathrm{~K}$.

A BSCCO 2212 tube of $70 \mathrm{~mm}$ diameter has been supplied by Hoechst (Frankfurt, DE). The sample, $250 \mathrm{~mm}$ long, has integrated silver contacts at the two ends where are soldered the copper end-caps. The rest of the length is coated with a thin layer of silver-gold alloy (about $21 \mathrm{~mm}^{2}$ cross section) onto which is soldered a metallic shunt acting as a current by-pass during quench. Previous measurements on a tube coated with pure silver resulted in high heat conduction.

Four YBCO bars, $200 \mathrm{~mm}$ long, have been supplied by Haldor Topsøe (Lyngby, DK). The bars, which are silvergold coated, are protected in case of resistive transition with a metallic shunt connected in parallel to the HTS.

Both the samples have been able to withstand a resistive transition without damaging. The results of the measurements are summarised in Table I. 
TABLE I

QUENCH MEASUREMENTS ON CLADDED HTS BULK SAMPLES

\begin{tabular}{llll}
\hline & & BSCCO cylinder & YBCO bars \\
\hline $\mathrm{I}$ & $\mathrm{A}$ & 12000 & 12000 \\
$\mathrm{Q}$ & $\mathrm{W}$ & $\sim 5$ & $\sim 2$ \\
$\mathrm{R}_{\mathrm{W}}$ & $\mathrm{n} \Omega$ & 35 & 20 \\
$\mathrm{R}_{\mathrm{C}}$ & $\mathrm{n} \Omega$ & 12 & 1 \\
$\mathrm{I}_{\mathrm{Q}}$ & $\mathrm{A}$ & 8000 & 12000 \\
$\mathrm{~T}_{\mathrm{Q}}$ & $\mathrm{K}$ & 83 & 85 \\
$\mathrm{U}_{\mathrm{MAX}}$ & $\mathrm{V}$ & 0.12 & 0.365 \\
\hline
\end{tabular}

$I=$ maximum current attained; $Q=$ heat conducted into the liquid helium at zero current; $R_{w}=$ contact resistance at the warm end of the HTS; $R_{C}=$ contact resistance at the cold end of the HTS; $\mathrm{I}_{\mathrm{O}}=$ current carried by the sample during the resistive transition; $\mathrm{T}_{\mathrm{Q}}=$ temperature measured at the warm end when the voltage across the HTS reaches about $1 \mathrm{mV} ; \mathrm{U}_{\mathrm{MAX}}=\operatorname{maximum}$ voltage measured across the HTS sample. This table supplements information given previously and referred to in the text.

Tests have been performed on HTS samples carrying $600 \mathrm{~A}$ between $1.8 \mathrm{~K}$ and $70 \mathrm{~K}$ in a test facility built at CERN [3]. Measurements on BSCCO 2223 PIT tapes (ASC, Westborough MA, IGC, Latham NY), BSCCO 2223 AFM samples (Enel, Milano I) BSCCO 2212 MCP rods (Hoechst DE) and YBCO 123 MTG rods (ATZ Adelwitz DE) have proven the viability of HTS components in 600 A leads: current capability and low heat conduction are met by all these materials. However, also for this low current, electrical contact at the two ends of the HTS must be carefully made to avoid spoiling the thermal performance due to high heat generation.

\section{COOLING METHOD}

HTS leads, made of an upper resistive stage and a lower superconducting stage, benefit from the low thermal conductivity and the ideally zero resistivity of the High Temperature Superconductor to reduce the heat conduction into the liquid helium and the consumption in liquefaction power. The total saving in cooling power depends on the type of cooling adopted for the resistive part of the lead [1], [4], [5], taking into account the exergy loss by evaporation of liquid helium and from gas heating.

Previous analyses have shown advantages and drawbacks of possible cooling methods within the fixed frame of the cryogenic system [6]. By cooling the resistive part of the lead with $20 \mathrm{~K}$ and $0.13 \mathrm{MPa}$ helium gas, recovered from the return lines of the beam screen, the cryogenic system benefits from a potential saving of cooling power of about $30 \%$ with respect to conventional leads. In addition, the availability of $20 \mathrm{~K}$ helium gas allows to fix the temperature at the warm end of the superconductor at values below $50 \mathrm{~K}$, more favourable for the HTS material in terms of current density and sensitivity to magnetic field.

The resistive part of the lead, cooled by forced flow of helium gas entering at $20 \mathrm{~K}$, is hydraulically separated from the superconducting part which operates in self cooling conditions. A jacket around the HTS forces the evaporated helium to cool by convection the HTS part of the lead before being released into the gaseous environment above the bath.

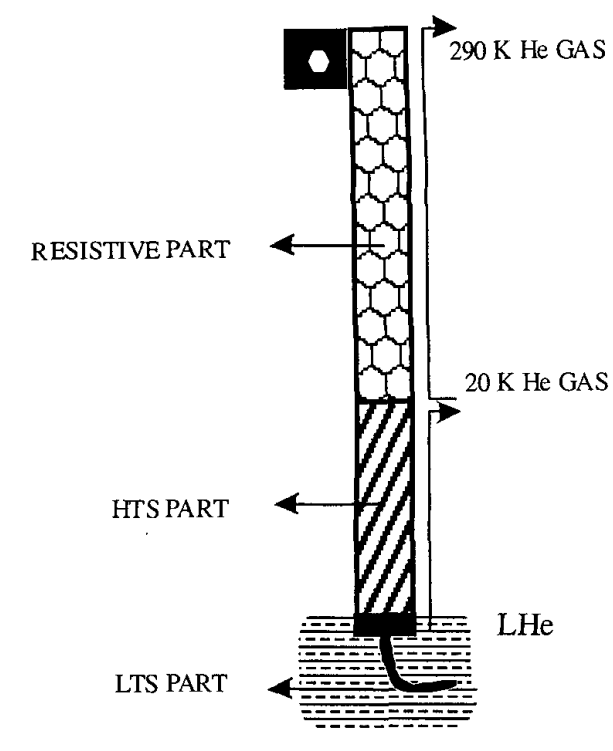

Fig. 2. Cooling scheme of the Large Hadron Collider HTS leads.

The temperature at the warm end of the superconductor is controlled by the $20 \mathrm{~K}$ helium mass flow. The cooling scheme of the HTS leads is sketched in Fig. 2.

Increasing the coolant flow, the temperature at the warm end of the superconductor decreases, reducing the amount of heat dissipated into the liquid helium. The balance between these two parameters allows to find a minimum in the cooling power, which corresponds to operation with a temperature at the warm end of the superconductor of about $50 \mathrm{~K}$ [4].

In Fig. 3 is represented the dependence of the heat dissipation at $4.5 \mathrm{~K}$, given by the sum of thermal conduction and of the ohmic generation at the cold electrical contact of the HTS, and of the $20 \mathrm{~K}$ coolant flow on the temperature at the warm end of the superconductor. The figure refers to a 13000 A lead whose resistive part is made of phosphorous deoxidized copper (PDC). The superconducting part, made of BSCCO 2223 tapes, is designed to withstand the current, in case of resistive transition of the HTS, during the unloading time of the magnets (time constant equal to 120 seconds).

Calculations have been made on such a type of current lead, defining the optimum geometry of a resistive heat exchanger connected to a chosen superconducting element [7]. The optimization is based on the numerical solution of the heat balance equation on the lead, taking into account the temperature dependence of the properties of the material and gas. The geometry of the normal conducting part depends on the properties of the material used for the heat exchanger and on the temperature at the warm end of the superconductor, the latter being a function of the $20 \mathrm{~K}$ helium mass flow, as shown in Fig. 4. The optimum geometry, for a given current, corresponds to the minimum flow which gives a stable temperature profile of the lead, reaching room temperature at the warm terminal with an ideally zero slope.

If the lead is operated with a mass flow lower than the 
TABLE II

DESIGN PARAMETERS OF HTS LEADS

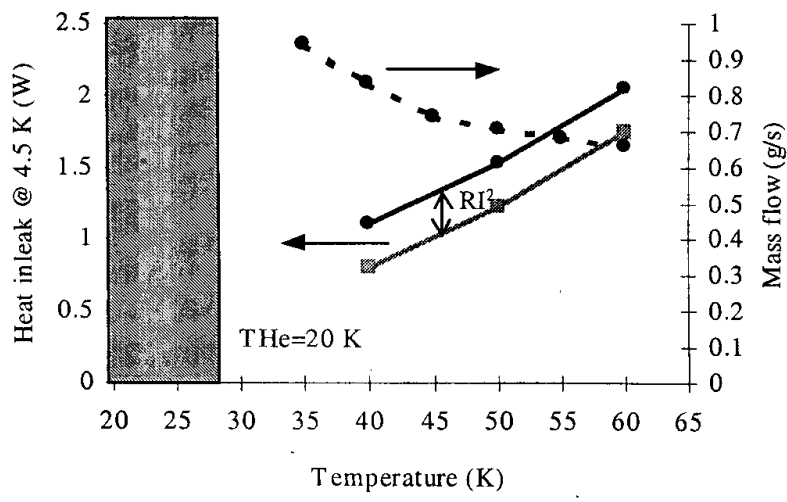

Fig. 3. Dependence of the $20 \mathrm{~K}$ helium mass flow and of the heat dissipation into the liquid helium from the temperature at the warm end of the superconductor for a 13000 A HTS lead.

optimum one, the temperature at the warm end of the superconductor increases together with the voltage across the resistive heat exchanger: below a critical flow, the lead would suffer a thermal run-away. For a mass flow higher than the optimum one, the lead would operate in over-cooled conditions, wasting power and risking icing.

In standby operation and for currents below the nominal, with a reduced mass flow which keeps the temperature at the warm end of the superconductor constant, the room temperature at the warm end of the lead is guaranteed by introducing power from a heater incorporated into the warm terminal. In this way condensation and ice build-up are avoided.

\section{HIGH-CURRENT LEADS}

On the basis of a theoretical analysis and of the encouraging test results, CERN has sent out to industry calls for tenders concerning $600 \mathrm{~A}$ and $13000 \mathrm{~A}$ HTS current leads [8]. Technical specifications have been written to define the performance, design and tests of these devices. The materials used for the resistive and for the superconducting part of the lead are, at this stage, chosen by the manufacturers on the basis of the specified technical requirements and their particular expertise.

The main lead parameters are listed in Table II. The leads must be designed for optimum thermal characteristics and good electrical, hydraulic and mechanical reliability. The limiting overall maximum dimensions are imposed by the infrastructure of the LEP tunnel inside which the feedboxes containing the leads will be installed.

In case of accidental irreversible transition of the HTS section to the resistive state, the leads must be able to withstand the current, without damage, during the unloading time of the magnets. The worst condition is represented by the leads powering the dipole circuits, where the

\begin{tabular}{llll}
\hline $\mathrm{I}$ & $\mathrm{A}$ & $13000 \mathrm{~A}$ & $600 \mathrm{~A}$ \\
\hline $\mathrm{d} \mathrm{d} / \mathrm{dt}$ & $\mathrm{A} / \mathrm{s}$ & $\leq 120$ & $\leq 5000$ \\
$\mathrm{U}$ & $\mathrm{kV}$ & 3.5 (He gas $)$ & 1.1 (He gas) \\
$\mathrm{m}(13000 \mathrm{~A})$ & $\mathrm{g} / \mathrm{s}$ & $<1$ & $<0.04$ \\
$\mathrm{~m}(0 \mathrm{~A})$ & $\mathrm{g} / \mathrm{s}$ & $<0.6$ & $<0.024$ \\
$\mathrm{THe}$ & $\mathrm{K}$ & 20 & 20 \\
$\mathrm{pHe}$ & $\mathrm{MPa}$ & 0.13 & 0.13 \\
$\mathrm{~T}$ & $\mathrm{~K}$ & $<50$ & $<50$ \\
$\mathrm{p}$ & $\mathrm{kPa}$ & $<5$ & $<5$ \\
$\mathrm{Q}(13000 \mathrm{~A})$ & $\mathrm{W}$ & $<1.5$ & $<0.08$ \\
$\mathrm{Q}(0 \mathrm{~A})$ & $\mathrm{W}$ & $<1$ & $<0.07$ \\
$\mathrm{Rw}$ & $\mathrm{n} \Omega$ & $<20$ & $<1000$ \\
$\mathrm{Rc}$ & $\mathrm{n} \Omega$ & $<2$ & $<30$ \\
$\mathrm{~L}$ & $\mathrm{~m}$ & $\leq 1.5$ & $\leq 1.5$ \\
$\Phi$ & $\mathrm{m}$ & $\leq 0.12$ & $\leq 0.03$ \\
\hline
\end{tabular}

$\mathrm{dL} / \mathrm{dt}=$ maximum unloading rate; $\mathrm{U}=$ rated insulation voltage to ground; $\mathrm{m}(13000 \mathrm{~A})=20 \mathrm{~K} \mathrm{He}$ mass flow at nominal current; $\mathrm{m}(0 \mathrm{~A})=20 \mathrm{~K}$ He mass flow at zero current; THe=inlet temperature of the $\mathrm{He}$ gas; $\mathrm{pHe}=$ inlet pressure of the $20 \mathrm{~K} \mathrm{He} ; \mathrm{T}=$ maximum operating temperature of the HTS; $\Delta \mathrm{p}=$ pressure drop of the $20 \mathrm{~K} \mathrm{He}$ mass flow; $\mathrm{Q}(13000 \mathrm{~A})=$ heat load at $4.5 \mathrm{~K}$ with nominal current; $\mathrm{Q}(0 \mathrm{~A})=$ heat load at $4.5 \mathrm{~K}$ with zero current; $\mathrm{Rw}=$ contact resistance at the warm end of the HTS; $\mathrm{R} c=$ contact resistance at the cold end of the HTS; L=overall length of the lead; $\Phi=$ external diameter of the lead.

current, during the discharge, decays exponentially with a time constant of 120 seconds. For this reason, particular efforts have been put in the study and in the measurement of the quench behaviour of different HTS samples.

To meet this requirement, the superconductor needs to be protected by a shunt, represented by the Ag-Au matrix of the BSCCO tapes or by an external element, connected in parallel to the HTS, designed to by-pass the current during a quench without spoiling the thermal performance. The cooling of the HTS part with the vapour generated by the HTS element itself at $4.5 \mathrm{~K}$ allows to keep the total heat load into the liquid helium within the specified values.

In order to test the prototype leads constructed by several manufacturers, proposing different HTS technologies, a cryostat has been built at CERN.

The 13000 A prototype leads have the HTS part made of various materials: PIT BSCCO $2223 \mathrm{Ag}-\mathrm{Au}$ tapes, using different percent of gold in the matrix, MCP BSCCO 2212, Dip Coated BSCCO 2212, AFM BSCCO 2223 and sintered YBCO 123.

The leads, supplied with all the instrumentation requested to characterise their thermal and electrical performance, are tested in pair with a short circuit at the cold end. The manufacturers of the $13000 \mathrm{~A}$ leads have been supplied with a bundle of low temperature superconducting wires free at the end to be connected by the supplier to the cold terminal of the HTS element. At the other end the wires are twisted and soldered onto a copper cylinder, silver-plated and machined to obtain a smooth and homogeneous surface. During testing, the two cylinders are clamped, face to face, onto two copper blocks housing low temperature superconducting wires along the current path. At $13000 \mathrm{~A}$, the contact resistance of this (two contacts) joint is considerably less than $2 \mathrm{n} \Omega$. In the 


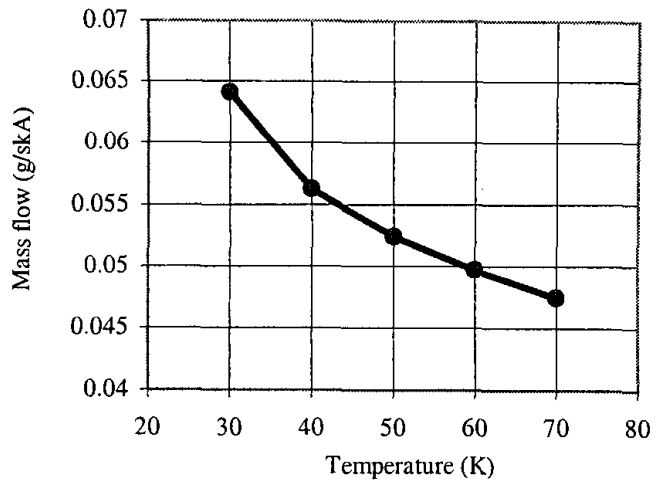

Fig. 4. Dependence of the $20 \mathrm{~K}$ helium mass flow on the temperature at the warm end of the superconductor.

LHC cryostats, each joint will be connected to the busbars going to the magnets.

The first pair of 13000 A prototype leads, supplied by Fuji Electric and recently tested at CERN, has met all the requirements of the technical specification. The test of the other prototypes will continue in the next months.

The prototype $600 \mathrm{~A}$ current leads will be tested at CERN in Spring 1999.

\section{LOW-CURRENT LEADS}

The powering of the corrector magnets will be done via local power converters. The leads, about 2000 in total, will operate at maximum currents of 60 or $120 \mathrm{~A}$.

The design philosophy asks for leads which are simple and have no valves or piping for gas return lines, thus avoiding any control problems. Besides being adapted to fit the space available, they must introduce a low heat inleak into the cryogenic system.

It has been decided, both for reasons of engineering difficulty and because of the potential saving in heat load is small, that the corrector leads will not contain HTS sections. For such low currents, reasonably low heat inleaks can be reached by using conventional leads.

The design of the $60 \mathrm{~A}$ current lead consists of a pure conduction cooled copper wire operating, in the insulation vacuum of the cryostat, between room temperature and the $1.9 \mathrm{~K}$ liquid helium bath. To minimise the heat dissipated into the liquid, the conductor is thermalized at two intermediate temperature levels. A first heat sink is provided by the $50 \mathrm{~K}$ $75 \mathrm{~K}$ helium gas, which cools the LHC thermal shield sheltering the cold mass from the ambient heat inleaks, and a second heat sink is cooled by the $20 \mathrm{~K}$ helium gas from the LHC beam screens. In this way, only the heat dissipated between $20 \mathrm{~K}$ and $1.9 \mathrm{~K}$ is conducted into the liquid helium. The thermalization is done by clamping the insulated conductor against the helium tubes operating for the leads as intermediate heat exchangers.
The lead cross section is optimised in each of the three different operating regions, in order to minimise the heat adsorbed at intermediate temperatures, while the length is imposed by the physical path the conductor has to follow inside the cryostat. The heat dissipated by an optimised conduction cooled lead operating at nominal current corresponds to: 0.046 W/A @ 50 K, 0.0072 W @ $20 \mathrm{~K}$, 0.0031 W/A @ $1.9 \mathrm{~K}$. These figures are, in reality, conservative for the corrector leads: the magnets will not operate constantly at the maximum rate, since the currents follow a gaussian distribution with a standard deviation of $26 \%$ of the maximum strength.

A cryostat has been built at CERN and tests of prototypes are in progress to evaluate the thermal and electrical performance of the corrector leads.

\section{CONCLUSION}

Thanks to the progress in HTS technology over the past years, it should soon be possible to make reliable high current leads incorporating HTS sections.

The LHC provides a perfect opportunity for the use of such leads, with its relatively high total current to pass from room temperature to the liquid helium environment and a particularly well adapted cryogenic system which allows to cool the warm end of the HTS section to a temperature lower than $50 \mathrm{~K}$.

The test of the first pair of 13000 A HTS prototype leads gave very encouraging results, performing according to the strict requirements of the technical specification. Measurements of several prototypes will allow to compare different technologies in order to choose the most suitable for the LHC machine.

\section{REFERENCES}

[1] A. Ballarino, A. Ijspeert, M. Teng, U. Wagner, S. Harrison, K. Smith, L. Cowey, "Design of $12.5 \mathrm{kA}$ current leads for the Large Hadron Collider using High Temperature Superconductor material", ICEC I6 proceedings, pp. 1143-6, 1996.

[2] M. Teng, A. Ballarino, R. Herzog, A. Ijspeert, C. Timlin, S. Harrison, K. Smith, "Evaluation of HTS samples for 12.5 kA current leads", Applied Superconductivity 1997, vol. 2, pp. 1203-6, 1997.

[3] A. Ballarino, A. Ijspeert, "Design and tests on the 30 to 600 A HTS current leads for the Large Hadron Collider", ICEC 16, pp. 1147-50, 1996.

[4] A.Ballarino, "Discendenti di corrente raffreddati ad elio ed utilzzanti materiale superconduttore ad alta temperatura critica", $15^{\text {th }} U I T$ National Heat Transfer Conference, pp. 971-979, 1997.

[5] A. Ballarino, A. Ijspeert, "Expected advantages and disadvantages of High-Tc current leads for the Large Hadron Collider orbit correctors", Advances in Cryogenic Engineering, vol. 39, 1994.

[6] A. Ballarino, A. Ijspeert, U. Wagner, "Potential of High Temperature superconductor current leads for LHC cryogenics", ICEC I6 proceedings, 1996.

[7] A. Ballarino, "Discendenti di corrente realizzati con materiale superconduttore ad alta temperatura critica per l'alimentazione dei magneti del Large Hadron Collider", $P h D$ Thesis, Politecnico di Torino, Torino, 1997.

[8] T. Taylor, "High Temperature Superconducting Leads for the Large Hadron Collider", ASC-98, 1998. 